\title{
Oral cavity health among cystic fibrosis patients: Literature overview
}

\author{
Katarzyna Herman ${ }^{1, A, D-F}$, Małgorzata Kowalczyk-Zając ${ }^{1, A, D, E}$, Tomasz Pytrus ${ }^{2, A, D, E}$ \\ ${ }^{1}$ Department of Conservative Dentistry and Pedodontics, Wroclaw Medical University, Poland \\ ${ }^{2} 2^{\text {nd }}$ Department of Pediatrics, Gastroenterology and Nutrition, Wroclaw Medical University, Poland \\ A - research concept and design; $\mathrm{B}$ - collection and/or assembly of data; $\mathrm{C}$ - data analysis and interpretation; \\ $D$ - writing the article; $E$ - critical revision of the article; $F$ - final approval of article
}

\section{Katarzyna Herman \\ E-mail: ksherman@wp.pl \\ Funding sources \\ None declared \\ Conflict of interest \\ None declared}

Address for correspondence

Received on March 1, 2016

Revised on May 15, 2016

Accepted on July 5, 2016

\begin{abstract}
Cystic fibrosis is a genetic disorder in which the mutation of the Cystis Fibrosis Transmembrane Conductance Regulator (CFTR) gene that codes the protein forming a chloride channel of epithelial cells results in its distorted functioning. The manifestations of the disorder are mainly observed in the respiratory and digestive system. Accumulation of sticky and thick mucus is the dominant clinical symptom; it leads to chronic infections and gradual tissue destruction. Although cystic fibrosis remains incurable, it is currently feasible to extend patients' life expectancy thanks to modern therapy possibilities. As cystic fibrosis is no longer the domain of pediatricians, health care to CF patients needs to be provided by doctors of various specializations. The multidisciplinary team of doctors should include a dentist aware of specific prevention and treatment needs of this group of patients. It results from the fact that in the course of cystic fibrosis it is possible to observe a variety of changes in the oral cavity environment. The study presents dental issues observed in CF patients and reported in literature. Particular attention was paid to dental caries, mineralization disorders of hard dental tissues, gingivitis and the change in the content and properties of saliva; moreover, prevention and treatment options regarding oral cavity health is this group of patients were taken into consideration.
\end{abstract}

Key words: cystic fibrosis, gastrointestinal disorders, oral disease, genetic markers

DOI

10.17219/acem/64054

\section{Copyright}

Copyright by Author(s)

This is an article distributed under the terms of the

Creative Commons Attribution Non-Commercial License

(http://creativecommons.org/licenses/by-nc-nd/4.0/) 
Cystic fibrosis is a genetic inherited disorder in an autosomal recessive manner and caused by mutations within the CFTR protein coding gene (Cystic Fibrosis Transmembrane Conductance Regulator) on the long arm of chromosome 7. It is a systemic disease with different clinical expression. Fully symptomatic cystic fibrosis is characterized by bronchitis, pneumonia, exocrine pancreatic insufficiency (EPI), and a pathognomonic increase in the concentration of $\mathrm{Cl}^{-}$in sweat. Patients affected by the disease show many symptoms in other organs. In many cases no pancreatic insufficiency is observed. The length and quality of life is usually determined by the changes in the respiratory system. The basic genetic defect leads to the production of thick, sticky mucus and its accumulation, which results in chronic infections and gradual destruction of tissues and organs.

Apart from the changes typical for fully symptomatic cystic fibrosis, numerous changes in the oral cavity may occur as well. This study presents dental problems experienced by cystic fibrosis patients and reported in the literature. Special focus was placed on caries, mineralization disorders of hard tissue, gingivitis, and changes in the content and properties of saliva. Among a multidisciplinary team of doctors that take care of a cystic fibrosis patient, there should be a dentist whose awareness of the specific prevention and treatment needs in this group of patients will make it possible to provide comprehensive treatment.

\section{Introduction}

Cystic fibrosis (lat. mucoviscidosis) is the most frequent genetically conditioned total exocrine dysfunction among Caucasians. It is inherited in an autosomal recessive manner and the frequency of its occurrence (1:2000 births) is similar for both sexes. ${ }^{1-3}$ Since 2006 , due to the high population frequency of the disease, two-stage newborn screening for cystic fibrosis has been conducted in Poland. First, the level of trypsin in the blood is determined, and then a molecular test is conducted. Immunoreactive tripsin (ITR) is measured in a blood sample drawn on a piece of absorbent paper between the $3^{\text {rd }}$ and the $6^{\text {th }}$ day of life. An increased level of ITR may indicate cystic fibrosis; the initial diagnosis is later confirmed with a molecular DNA analysis. With the use of this test, it is possible to establish an early diagnosis and implement professional treatment.

The disease is caused by mutations of the CFTR gene, which can be found on the long arm of chromosome 7 (7q31.2), and is responsible for coding protein with the same name. The protein constitutes the apical membrane chloride channel of the epithelioid cells. The structure of the channel consists of several domains which perform specific functions: the endothelial domains set the pathway of chloride ions through the cell membrane, and the regulatory domains are responsible for opening and closing the channel. The process is connected with phosphorylation of protein by cAMP (cyclic adenosine monophosphate) dependent protein kinase. ${ }^{4}$ The functioning of the chloride channel is conjugated with the activity of other epithelioid ion channels and transporters. Therefore, it indirectly regulates the flow of carbonate, sodium and potassium ions. ${ }^{5-7}$ So far, over 1600 mutations of the CFTR gene have been reported. They all result in impairment of the structure and functioning of the chloride channel, which in turn leads to disturbance of the general ion balance of the epithelium.

The most common mutation type diagnosed in $90 \%$ of cases is deletion of phenylalanine 508 ( $\Delta$ F 508 mutation). ${ }^{7}$ The $\triangle F 508$ mutation of the CFTR gene that causes CF is diagnosed in patients from Northern Europe with a frequency of $75-88 \%$, and in Poland with a frequency of $53-73 \%$. Currently, it is known that apart from classic gene mutations, the phenotype is also determined by polymorphic changes in the CFTR gene. Furthermore, there are complex mutations, i.e. 2 mutations, in a single allele.

Organs which contain epithelium accumulate thick and sticky mucus that is very difficult to remove. This, in turn, leads to chronic inflammation and gradual destruction of tissues. Changes in the respiratory and digestive system are dominant in a majority of patients. The mucus accumulated in the area of the bronchial tree stimulates proliferation of pathogens, particularly Staphylococcus aureus, Pseudomonas aeruginosa and Haemophilus influenzae, which leads to severe chronic infections the complications of which are the most common cause of death.,2,4 The fundamental cause of death is development of uneven respiratory insufficiency which may cause death in the first decade of life in the group of untreated cases. ${ }^{2}$

In most cases (85-90\%), there are also inflammatory/ fibrotic lesions of the pancreas that lead to impairment of its exocrine function. ${ }^{8}$ As a result of decreased secretion of the pancreatic juice, digestion and secondary intestinal absorption is disturbed, and this leads to chronic steatorrhea. Its consequences include malnutrition, deficiencies in vitamins (particularly ADEK) and trace minerals, and impaired growth, as well as disturbed bone mineralization in the form of osteopenia and osteoporosis. Some patients, especially those who have suffered from the disease for a long time, also have severe diabetes that is very difficult to control. It results from impaired endocrine function and damage to the pancreatic islets apparatus. Other symptoms connected with the digestive system include meconium ileus which can be observed in the first days of life and may lead to perforation and meconium peritonitis.

Malfunction of the bile ducts in the form of intrahepatic cholestasis leads to inflammation, development of cirrhosis and progressive failure of the organ which in consequence requires a liver transplant. Other complications connected with liver function include increased 
occurrence of cholelithiasis which results from abnormal metabolism of bile acid.,4 Other conditions related to the digestive system include intensified gastroesophageal reflux, and the so-called equivalents of meconium ileus that afflict older children. Gastroesophageal Reflux Disease (GERD) has a complex pathogenesis, and its intensification may directly impact the progression of bronchopulmonary dysplasia. ${ }^{9}$ Increased pain and flatulence may be caused by Distal Intestinal Obstruction Syndrome (DIOS). Partial DIOS is diagnosed when the fecal mass is retained in the ileocecal area. Complete DIOS is characterized by a co-occurrence of bile stained vomiting and/or increased levels of fluid in the small intestine on a plain abdominal X-ray.

Other common pathognomonic symptoms of the disease include rectal prolapse which had constituted a significant diagnostic indicator before screening tests came into use. Moreover, there are reports of an increased risk of gastrointestinal carcinoma. ${ }^{10}$ Cystic fibrosis patients may also suffer from infertility which predominantly concerns men (as a result of ejaculatory duct obstruction), as well as from vasculitis and arthritis. Fertility-related dysfunctions among men pose a grave problem among adults suffering from cystic fibrosis, and before screening tests were introduced, they had served as an indication that tests towards CF should be done, and as a result, late diagnosis was established. There are also characteristic changes in the composition of sweat which becomes abnormally salty as a result of increased concentration of chloride ions. ${ }^{4}$

In CF diagnosis, in addition to the aforementioned screening test, a sweat test is used which shows a significantly high concentration of chloride anions in sweat $\left(\mathrm{Cl}^{-}>60 \mathrm{mmol} / \mathrm{L}\right.$, in newborns $\left.>40 \mathrm{mmol} / \mathrm{L}\right)$, in at least 2 independently done tests. As an alternative to the sweat test, a conductometric test that analyses the electrical conductivity of sweat is done. Should diagnostic difficulties arise (i.e. inconclusive results of the sweat test, lack of two mutations of the CFTR gene), the transepithelial nasal potential difference may be measured. The ultimate confirmation of the diagnosis is a molecular analysis and detection of one of the disease specific mutations. Exocrine pancreatic insufficiency can be confirmed by low concentration of fecal elastase-1.

The treatment of CF is interdisciplinary and it mainly concerns the respiratory and digestive system. The therapy of respiratory complications is based on mucolytics, carefully selected antibiotics, aerosol therapy and kinesitherapy.

Gastrointestinal treatment is mainly based on nutrition intervention, supplementation with pancreatic enzymes, administration of vitamins and macro- and microelements the role of which is to prevent malnutrition and disturbed bone mineralization. Therapy with pancreatic enzymes is particularly important. Enzymatic supplementation is necessary when clinical symptoms of exocrine pancreatic insufficiency, i.e. chronic diarrhea, particularly steatorrhea, and small body weight increase, have been observed. The dose of pancreatic enzymes is considered to be proper when it leads to decreased loss of fat with stool (normalization of stool frequency and type), elimination of flatulence and abdominal pain, and continual increase in the body weight.

Although cystic fibrosis remains incurable, progress in the therapy has resulted in significant prolongation of patients' lifespan. Several decades ago, most CF patients died in the first decade of life. ${ }^{4}$ Currently, average life expectancy is 37 years; therefore, cystic fibrosis is no longer the sole domain of pediatricians. ${ }^{1,2}$ Multidisciplinary medical care helps ensure the best possible comfort of life. This should also include dental treatment and preventive dental care, since this group of patients may demonstrate specific treatment needs.

The aim of the study is to highlight dental problems reported in current literature among patients suffering from cystic fibrosis.

\section{Dental caries}

The presence of sugars in the oral cavity which are delivered mainly with food is a necessary condition for the development of dental caries. Carbohydrates are used by cariogenic bacteria, particularly Streptococcus mutans and Lactobacillus acidophilus, for metabolic processes. As a result of these transformations, the $\mathrm{pH}$ of the environment is reduced below the critical values, and the demineralization process of the enamel may begin. The consequence of this process is carious lesions. ${ }^{7}$ The most negative effects are observed as a result of even small amounts of carbohydrate substrate.

When dealing with CF patients, special attention needs to be paid to the presence of risk factors related to dental caries. Exocrine pancreatic insufficiency and malabsorption necessitate constant supplementation with pancreatic enzymes, and following a special diet. Daily calorie requirement in the case of CF is $130-150 \%$ of the regular requirement for healthy adults. ${ }^{11}$ It is met by consuming high-energy meals, usually rich in fat, carbohydrates and protein. ${ }^{12}$ It is also recommended to use supplements frequently containing glucose polymers which break down in the oral cavity to monosaccharides. The supplements are typically used in the form of drinks or powders added to dishes. ${ }^{13}$ Young children take medication in the form of sweet syrups or suspensions which, if administered frequently and over long periods of time, may cause the development of carious lesions, especially in upper primary teeth. ${ }^{14}$ Aerosol medications characterized by acid reaction and containing sugar may also be a potential risk factor in the development of dental caries. ${ }^{3}$ Special attention is also paid to overprotective parents who focus on the primary disease and simultaneously 
disregard oral health, neglect oral hygiene and allow too frequent consumption of sweets. ${ }^{13}$ Despite the risk factors of the development of dental caries observed in the course of CF, reports on the issue presented in the literature are inconclusive.

Laboratory tests have shown drastic development of dental caries and fast destruction of dental crowns among mice with the $\Delta \mathrm{F} 508$ mutation after only several weeks of being fed a cariogenic diet. At the same time, a significant increase in the bacterial colony of Streptococcus mutans and a decrease in the concentration of hydrocarbonate ions in the saliva have been observed. Impaired function of this main buffering system and a resulting decrease in the $\mathrm{pH}$ of the saliva significantly influence the rapidly progressing enamel demineralization process. ${ }^{7}$ Certain clinical research seems to confirm an increased risk of dental caries among CF patients. Olejniczak et al. determined a significantly more frequent occurrence of dental caries in the permanent teeth of children aged 6-12 and suffering from cystic fibrosis. ${ }^{11}$ However, in a group of patients aged 1-5, there was a reverse tendency. Moreover, there were no significant differences observed in a group of adolescents aged above 13. Similar results were obtained by Dąbrowska et al. ${ }^{15}$ Peker et al., in turn, did not report any differences in a group aged $3-12 .^{3}$

Many authors have emphasized the existence of an interesting phenomenon - a less frequent occurrence of dental caries in CF patients in comparison with healthy subjects. ${ }^{16-23}$ According to Kinirons's reports, there is a significantly lower level of dental caries in primary teeth, and a similar yet less strongly marked tendency in permanent teeth. ${ }^{16}$ Ferrazzano et al. have confirmed a similar, statistically significant dependency in both primary and permanent teeth in children aged $7-12 .{ }^{20}$ According to Aps et al., a significantly higher level of dental caries has been reported in the CF of homozygotes in comparison with healthy ones and heterozygotes. ${ }^{21}$ When comparing the dental status of children with CF aged 2.5-16.5 with children suffering from other chronic diseases of the respiratory tract, Narang et al. also observed a certain tendency to a less frequent occurrence of dental caries in the cases of cystic fibrosis. ${ }^{12}$ Researchers tend to hold the view that the aforementioned phenomenon may result from more frequent and long-term antibiotic therapy, which reduces the titer of cariogenic pathogens in the oral cavity. ${ }^{18,24}$ There are reports of a significantly lower level of Streptococcus mutans among patients suffering from cystic fibrosis. ${ }^{22}$ The dependency between antibiotic therapy and the titer of cariogenic bacteria may be particularly important in small children, among whom Staphylococcus aureus is the dominant pathogen of the respiratory system. The infections it causes are treated with penicillin, which is also effective against streptococci that reside in the oral cavity. After the $10^{\text {th }}$ year of life, the microbiological profile changes; infections caused by Pseudomonas aeruginosa are dominant and they are treated with tobramycin, which is an aminoglycoside antibiotic and is not effective against cariogenic pathogens. As a consequence, it is believed that the risk of caries development among patients treated with this antibiotic may be higher. ${ }^{18,19}$

When analyzing the risk factors that influence the development of dental caries, the composition and properties of the saliva are also taken into consideration; however, the reports related to this issue are often inconclusive. In the course of cystic fibrosis, salivary secretion may be impaired and it may be caused by damage to the salivary glands, or it may be a side effect of the medications received as part of the treatment. Decreased salivary flow rate, and a simultaneous decrease in its buffering potential responsible for the acid alkaline balance, may lead to the occurrence of dental caries. ${ }^{15}$ However, there are also reports of significantly higher average $\mathrm{pH}$ values, buffer capacity and higher concentration of calcium and phosphorus ions in the saliva, which have a positive impact on maintaining the acid alkaline balance of the oral cavity environment among patients suffering from CF. ${ }^{17,22,25}$ It is thought that the increased capability of the salivary buffering systems may be connected with the supplementation of pancreatic enzymes administered to CF patients. ${ }^{24}$

\section{Disturbed mineralization of hard dental tissues}

Lesions in the enamel may appear in the course of chronic, incurable diseases as a symptom of the disturbed mineralization of teeth. It is a long-term process that takes place in the pre-eruptive period and is based on the gradual precipitation of mineral compounds on the organic matrix of tooth buds. In the primary teeth, the process starts before the baby is born. In the case of permanent teeth, the beginning of the mineralization process is set in the following way: molars - in the perinatal period, incisors and cuspids - in the $1^{\text {st }}$ year of life, premolars - between the ages of 2 and $2.5,2^{\text {nd }}$ molars - in the $3^{\text {rd }}$ year of life, and $3^{\text {rd }}$ molars - in the $8^{\text {th }}-10^{\text {th }}$ year of life. Clinical dysfunctions of this process manifest themselves in the form of fuzzy or confined enamel opacities or hypoplasia (areas of thinner enamel or complete lack thereof). Many etiological factors effecting in such lesions are known and they include, for example, other systemic diseases and medications they are treated with. ${ }^{26}$

Molar incisor hypomineralisation (MIH) is a special form of the described abnormality. It is defined as a developmental enamel dysfunction that involves 1 or more molars with frequently coexisting lesions in the incisors. Clinically, enamel opacities of various sizes and colors are observed. The enamel is soft and porous and tends to destroy easily. The etiology of MIH has not been conclusively explained. Apart from a genetic component, 
systemic diseases, medications (e.g. antibiotics) and environmental impact factors from early childhood, particularly occurring in the first 3 years of life, are taken into consideration as possible causes of the abnormality. ${ }^{18}$

Research conducted on laboratory animals has shown a connection between disturbances in ion balance related to the defect of the CFTR protein and the occurrence of disturbed enamel mineralization. ${ }^{27-30}$ Tuggle et al. have observed a change in the coloration and lack of physiological abrasion of incisors in rats devoid of the CFTR gene. Premature degeneration of ameloblasts resulting in altered firmness and coloration of the enamel has been observed in mice with CF. ${ }^{29}$ The aforementioned data is confirmed in certain clinical reports. ${ }^{23,31,32}$ Ferrezzano et al. have observed developmental enamel defects in $56 \%$ of the subjects with CF; the same concerned $22 \%$ of the healthy subjects. ${ }^{31} \mathrm{Hy}$ poplasia of the permanent tooth enamel (23\% in the CF patients vs $1.5 \%$ in the healthy subjects) was the most frequently observed dysfunction. A more frequent occurrence of disturbed mineralization in the case of $\mathrm{CF}$ has also been determined by Azevedo et al.; however, the statistical significance only concerned permanent teeth. ${ }^{32}$ The most frequently observed dysfunction was opacities. Similar data was obtained by Narang et al., who compared a group of patients suffering from CF with patients afflicted by other diseases of the respiratory system. ${ }^{11}$ However, no dependency between cystic fibrosis and molar incisor hypomineralisation has been confirmed. ${ }^{17}$

\section{Gingivitis}

In the literature, there are numerous reports indicating a lower risk of gingivitis coexisting with cystic fibrosis. ${ }^{15,21,23,32,33}$ Kinirons et al. have observed a significant negative correlation between antibiotic therapy applied for 4 weeks before the examination and both gingivitis and the amount of dental plaque in patients with CF. Olejniczak et al. have determined a statistically lower percentage of patients with gingival bleeding and dental calculus among patients aged 13-18 and 19-29 in comparison with healthy subjects. ${ }^{33}$ A similar tendency has been observed among children aged 6-16, yet with no statistical significance. Different data was obtained by Narang et al., who observed a more frequent, yet statistically insignificant, occurrence of dental calculus and gingivitis in CF patients in comparison with patients suffering from other chronic diseases of the respiratory system. ${ }^{12}$ Increased calculus accumulation among CF patients has also been reported by Kinirons. ${ }^{16}$ This phenomenon may result from changes in the concentration of calcium and phosphorous in the saliva and an increase in its $\mathrm{pH}$, which conduces precipitation of mineral salts and formation of hard deposits..$^{16,22}$

\section{Saliva}

In the course of cystic fibrosis, there is a series of significant changes in the composition and properties of saliva which are an expression of salivary gland dysfunction and a side effect of pharmacotherapy. In proper conditions, the primary secretion of salivary glands shows a concentration of sodium and chloride ions which is similar to plasma and interstitial fluid. While the secretion is moving along the excretory ducts, ions are absorbed by chloride (CFTR) and sodium $(\mathrm{ENaC})$ channels. ${ }^{7,35}$ As a result, the solution becomes hypotonic. The distorted function of the CFTR channel leads to increased concentrations of chloride, sodium and potassium ions in the saliva. A similar mechanism occurs in sweat glands; due to this fact, it is suggested that saliva may potentially be used for diagnosing cystic fibrosis as an alternative to the sweat test. ${ }^{34}$

Salivary flow rate is an essential parameter that conditions maintaining homeostasis of the oral cavity environment. Fast secretion results in efficient clearing and supplying ingredients that are necessary for proper functioning of the mucosa, periodontium and teeth. Salivary secretion is controlled by the autonomic nervous system. Experiments conducted on animals have shown a diminished response of the salivary glands to adrenergic stimulation in the course of CF. ${ }^{35}$ Certain clinical research has confirmed decreased salivary secretion in the case of CF; however, other authors have not determined such a dependence. ${ }^{36,3}$ Inconclusive reports also concern changes in the $\mathrm{pH}$ values and buffer capacity, which play a significant role in maintaining acid alkaline balance by regulating the demineralization and remineralization processes of hard dental tissues. Kinirons has reported a significant increase in average values of the aforementioned parameters among patients suffering from CF. ${ }^{17}$ In other reports, it has been determined that there is a reverse tendency - a significant decrease in $\mathrm{pH}$ levels and buffer capacity. $3,34,36$

In the course of cystic fibrosis, changes may also appear in the organic components of saliva. Salivary proteins play various roles in the oral cavity environment. They protect the mucosa against irritative factors, initiate the formation of the dental pellicle that protects the enamel, facilitate the process of mastication and swallowing, and following carbonates and phosphates, constitute the third buffering system that controls the $\mathrm{pH}$. There is a significant increase in protein concentration among patients suffering from $\mathrm{CF}$ which has been observed by Aps et al., Modesto et al. and Livnat et al. ${ }^{21,36,37}$ Peker et al., in turn, have not confirmed such a connection. Sialic acid, which is a component of glycoproteins, performs a protective role for the tissues of the oral cavity. By covering the oral mucosa, it protects it against dryness, damage and bacterial penetration. Impaired formation of glycoproteins may be one of the causes of bacterial colonization in the respiratory tract among patients 
suffering from CF. Modesto et al. have identified a significant decrease in the concentration of total, free and combined sialic acid in the saliva of cystic fibrosis patients in comparison with healthy subjects. ${ }^{36}$ According to the authors, the cause of this phenomenon may lie in using mucolytics which, on the one hand, facilitate clearing the mucus, yet on the other hand, may simultaneously impair proper formation of glycoproteins.

In the course of cystic fibrosis, a significant decrease in the enzymatic activity of salivary $\alpha$-amylase and the peroxidase system has been demonstrated. ${ }^{36-38}$ The former enzyme initiates the digestion of starch, and it is also capable of bonding with bacteria and the pellicle. As a result, it may have an impact on the carious process. ${ }^{38}$ The latter, i.e. the salivary peroxidase system, is one of the innate immune systems that function in the oral cavity. In the presence of peroxidase and $\mathrm{H}_{2} \mathrm{O}_{2}$, thiocyanates $\left(\mathrm{SCN}^{-}\right)$that naturally occur in the saliva are oxidized to hypothiocyanites, which demonstrate antibacterial, antiviral and antifungal properties. A decreased activity of this system observed among patients suffering from cystic fibrosis is probably related to impaired secretion of thiocyanates which is dependent on the CFTR channel. ${ }^{38}$

The latest reports also demonstrate a significantly decreased activity of thromboplastin (factor III of the coagulation parameters) in the saliva, which may lead to delayed wound healing and predisposition to intense bleeding resulting from gingival inflammation. ${ }^{3}$

\section{Summary}

Cystic fibrosis is a systemic disease that may also have an impact on the functioning of the oral cavity environment. The occurrence of dental caries in the course of cystic fibrosis is a result of many factors demonstrating protective properties, yet at the same time also increasing the risk of the development of the disease. According to the American Academy of Pediatric Dentistry, children suffering from chronic diseases and demonstrating special health care needs, should constitute a high caries risk group. ${ }^{13}$ Most reports indicate a lower risk of gingivitis among patients suffering from CF; however, simultaneously, there is a stronger tendency for dental calculus formation. The role of the dentist will then be the regular removal of dental calculus. Changes in the composition and properties of saliva may result in impaired homeostasis of the oral cavity environment. Dysfunctions in the salivary flow rate, its $\mathrm{pH}$ and buffer capacity, as well as the impaired protective and antibacterial role of the saliva, increase the risk of inflammation of the mucosa and damage to hard dental tissues. Therefore, it is necessary to schedule regular dental check-ups, recommend the use of remineralizing and antibacterial rinses, and if needed, the use of artificial saliva products. It is also essential to raise the awareness of patients suffering from cystic fibrosis and their caregivers and inform them about potential problems that may occur in the oral cavity, as well as about possible treatment and prevention methods.

\section{References}

1. Walkowiak J, Pogorzelski A, Sands D, et al. Zasady rozpoznawania i leczenia mukowiscydozy: Zalecenia Polskiego Towarzystwa Mukowiscydozy 2009 Poznań - Warszawa - Rzeszów. Standardy Medyczne. 2009;6:352-378.

2. Cutting RG. Cystic fibrosis genetics: From molecular understanding to clinical application. Nat Rev Genet. 2015;16(1):45-56.

3. Peker S, Kargul B, Tanboga I, et al. Oral health and related factors in a group of children with cystic fibrosis in Istanbul, Turkey. Niger J Clin Pract. 2015;18(1):56-60.

4. Witt M, Majka L. Cystic fibrosis-a disease well studied but still mysterious. Alerg Astma Immunol. 1997;2(3):157-161.

5. Kloch M, Stanisławska J. Cystic Fibrosis. CEJSH. 2014;2:11-17.

6. Catalán MA, Nakamoto T, Gonzalez-Begne M, et al. Cftr and ENaC ion channels mediate $\mathrm{NaCl}$ absorption in the mouse submandibular gland. J Physiol. 2010;588(4):713-724.

7. Catalán MA, Scott-Anne K, Klein MI, et al. Elevated incidence of dental caries in a mouse model of cystic fibrosis. PLoS One. 2011;31;6(1):e16549.

8. Rosenstein BJ, Cutting GR. The diagnosis of cystic fibrosis: A consensus statement. Cystic fibrosis Foundation Consensus Panel. J Pediatr. 1998;132(4):589-595.

9. Dziekiewicz MA, Banaszkiewicz A, Urzykowska A, et al. Gastroesophageal reflux disease in children with cystic fibrosis. Adv Exp Med Biol. 2015;19 [Epub ahead of print].

10. Neglia JP, FitzSimmons SC, Maisonneuve P, et al. The risk of cancer among patients with cystic fibrosis. Cystic Fibrosis and Cancer Study Group. N Engl J Med. 1995;332(8):494-499.

11. Olejniczak M, Wierchoła B, Emerich-Poplatek K, Adamowicz-Klepalska B. Oral cavity ecology with respect to dental status and dental treatment needs in examined population of patients suffering from cystic fibrosis. Dent Med Probl. 2003;40(2):337-347.

12. Narang A, Maguire A, Nunn JH, Bush A. Oral health and related factors in cystic fibrosis and other chronic respiratory disorders. Arch Dis Child. 2003;88(8):702-707.

13. Moursi AM, Fernandez JB, Daronch M, Zee L, Jones CL. Nutrition and oral health considerations in children with special health care needs: Implications for oral health care providers. Pediatr Dent. 2010;32(4):333-342.

14. Maguire A, Rugg-Gunn AJ, Butler TJ. Dental health of children taking antimicrobial and non-antimicrobial liquid oral medication long-term. Caries Res. 1996;30(1):16-21.

15. Dąbrowska E, Błahuszewska K, Minarowska A, Kaczmarski M, Niedźwiecka-Andrzejewicz I, Stokowska W. Assessment of dental status and oral hygiene in the study population of cystic fibrosis patients in the Podlasie province. Adv Med Sci. 2006;51 (suppl 1):100-103.

16. Kinirons MJ. Dental health of patients suffering from cystic fibrosis in Northern Ireland. Community Dent Health. 1989;6(2):113-120.

17. Kinirons MJ. Increased salivary buffering in association with a low caries experience in children suffering from cystic fibrosis. J Dent Res. 1983;62(7):815-817.

18. Peker S, Mete S, Gokdemir Y, Karadag B, Kargul B. Related factors of dental caries and molar incisor hypomineralisation in a group of children with cystic fibrosis. Eur Arch Paediatr Dent. 2014;15(4): 275-280.

19. Chi DL. Dental caries prevalence in children and adolescents with cystic fibrosis: A qualitative systematic review and recommendations for future research. Int J Paediatr Dent. 2013;23(5):376-386.

20. Ferrazzano GF, Orlando S, Sangianantoni G, Cantile T, Ingenito A. Dental and periodontal health status in children affected by cystic fibrosis in a southern Italian region. Eur J Paediatr Dent. 2009;10(2):65-68.

21. Aps JK, Van Maele GO, Martens LC. Oral hygiene habits and oral health in cystic fibrosis. Eur J Paediatr Dent. 2002;3(4):181-187.

22. Aps JK, Martens LC. Oral health risks in patients with cystic fibrosis. Rev Belge Med Dent. 2004;59(2):114-120. 
23. Primosch RE. Tetracycline discoloration, enamel defects, and dental caries in patients with cystic fibrosis. Oral Surg Oral Med Oral Pathol. 1980;50(4):301-308.

24. Kinirons MJ. The effect of antibiotic therapy on the oral health of cystic fibrosis children. Int J Paediatr Dent. 1992;2(3):139-143.

25. Sorscher EJ, Breslow JL. Cystic fibrosis: A disorder of calcium-stimulated secretion and transepithelial sodium transport? Lancet. 1982;1(8268):368-370.

26. Cameron AC. Handbook of Pediatric Dentistry. $3^{\text {rd }}$ ed. Wroclaw: Elsevier Urban\&Partner; 2008:248-257, 458-459.

27. Bronckers AL, Lyaruu DM, Guo J, et al. Composition of mineralizing incisor enamel in cystic fibrosis transmembrane conductance regulator-deficient mice. Eur J Oral Sci. 2015;123(1):9-16.

28. Chang EH, Lacruz RS, Bromage TG, et al. Enamel pathology resulting from loss of function in the cystic fibrosis transmembrane conductance regulator in a porcine animal model. Cells Tissues Organs. 2011;194(2-4):249-254.

29. Tuggle KL, Birket SE, Cui X, et al. Characterization of defects in ion transport and tissue development in cystic fibrosis transmembrane conductance regulator (CFTR)-knockout rats. PLoS One. 2014;7:9(3):e91253.

30. Wright JT1, Kiefer CL, Hall KI, Grubb BR. Abnormal enamel development in a cystic fibrosis transgenic mouse model. J Dent Res. 1996;75(4):966-973.

31. Ferrazzano GF, Sangianantoni G, Cantile T, Amato I, Orlando S, Ingenito A. Dental enamel defects in Italian children with cystic fibrosis: An observational study. Community Dent Health. 2012;29(1):106-109.

32. Azevedo TD, Feijó GC, Bezerra AC. Presence of developmental defects on enamel in cystic fibrosis patients. J Dent Child (Chic). 2006;73(3):159-163.

33. Olejniczak M, Wierchoła B, Emerich-Poplatek K, Adamowicz-Klepalska B. Periodontal Status and Periodontal Treatment Needs among Patients Suffering from Cystic Fibrosis. Dent Med Probl. 2004;41(3)461-467.

34. Gonçalves AC, Marson FA, Mendonça RM, et al. Saliva as a potential tool for cystic fibrosis diagnosis. Diagn Pathol. 2013;8:46.

35. Droebner K, Sandner P. Modification of the salivary secretion assay in F508del mice - The murine equivalent of the human sweat test. J Cyst Fibros. 2013;12(6):630-637.

36. da Silva Modesto KB, de Godói Simões JB, de Souza AF, et al. Salivary flow rate and biochemical composition analysis in stimulated whole saliva of children with cystic fibrosis. Arch Oral Biol. 2015; 60(11):1650-1654.

37. Livnat G, Bentur L, Kuzminsky E, Nagler RM. Salivary profile and oxidative stress in children and adolescents with cystic fibrosis. J Oral Pathol Med. 2010;39(1):16-21.

38. Minarowski $Ł$, Sands D, Minarowska A, et al. Thiocyanate concentration in saliva of cystic fibrosis patients. Folia Histochem Cytobiol. 2008;46(2):245-246. 